\title{
Photoresponse Enhancement in Nanoscale Ge Photodetector through Split Bull's Eye Shaped Plasmonic Antenna
}

\author{
Fang-Fang Ren ${ }^{1,2 *}$, Kah-Wee Ang ${ }^{2}$, Mingbin Yu ${ }^{2}$, Guo-Qiang Lo ${ }^{2}$, Yi Shi ${ }^{1}$ \\ School of Electronic Science and Engineering, Nanjing University, Nanjing 210093, China \\ Institute of Microelectronics, A*STAR (Agency of Science, Technology and Research), Singapore 117685 \\ *Email: ffren@nju.edu.cn
}

\begin{abstract}
We exploit the idea of split bull's eye antenna for a nanometer-scale germanium photodetector operating at a standard communication wavelength of $1310 \mathrm{~nm}$. The nontraditional plasmonic metal aluminum has been implemented in the resonant antenna structure fabricated by standard CMOS processing. A significant enhancement in photoresponse is achieved in split bull's eye scheme due to an increased optical near-field in the active region.
\end{abstract}

\section{Introduction}

There exists an inherent trade-off between speed and quantum efficiency in a nanometer-scale germanium (Ge) photodetector metal-semiconductor-metal (MSM) photodetector. Bull's eye antennas provide an excellent strategy breaking the bottleneck by converting input light into long-range surface plasmon resonances (SPRs) and induce extremely strong optical intensity inside a small region. However, most of the reported antennas are not convenient for application due to the incompatibility of noble metals with the existing Si-CMOS technology [1]. Although the bull's eye antenna has the advantage of collecting optical signals from much wider area of illumination [2], but its larger antenna area limits the practical application for chip integration. To meet these two challenges, we exploit the novel design of nanometer-scale MSM Ge photodetector by splitting the conventional bull's eye antenna with a $\Phi$ shape nano-gap. The nontraditional plasmonic metal aluminum (Al) is chosen as antenna material for its CMOScompatibility [3]. Through careful theoretical design and experimental device fabrication, a significant photoresponse enhancement is achieved in split bull's eye scheme due to an increased optical near-field in the active region. With this novel antenna design, the effective grating area could be significantly reduced.

\section{Theoretical Design and Device Fabrication}

We consider three devices (A, B, and C) with different antennas for the wavelength of $1310 \mathrm{~nm}$. Fig. 1(a) and (b) show the top-view schematics for conventional and split bull's eye antennas, respectively. $\mathrm{Al}$ is chosen as the antenna material of metal layer with thickness of $h$. Devices A and B consist of conventional bull's eye antenna with SPR being present or absent at $1310 \mathrm{~nm}$ respectively. The parameters of the concentric grooves (depth $d$, width $b$, period number $P N$ on each side of the hole) and single hole (diameter $w$ ) are shown in Table 1. By splitting a bull's eye antenna into two halves with a $\Phi$-shape gap (width $g$ ), we form device $C$, which also supports SPR excitation around $1310 \mathrm{~nm}$. The two semi-circles in the $\Phi$-shape gap (Fig. 1(c)) can provide sharp corner tips for additional local optical resonances caused by field singularity when compared to the parallel-line design (Fig. 1(d)). The crosssectional views of these two antennas are similar as shown in Fig.1(e). The grating period $a$ is defined as the distance between centers of two nearest grating grooves, and also from the aperture center to the neighboring groove center. It is noted that the first tooth (counting from the center) has a different width of $a-0.5(b+w)$ from others $(a-b)$.

Table 1 Structural Parameters of Devices A, $\mathrm{B}$, and $\mathrm{C}$.

$\operatorname{device} a(\mathrm{~nm}) b(\mathrm{~nm}) d(\mathrm{~nm}) h(\mathrm{~nm}) w(\mathrm{~nm}) g(\mathrm{~nm})$

\begin{tabular}{lrrrrrr}
\hline A & 1290 & 200 & 100 & 400 & 550 & -- \\
B & 1450 & 400 & 100 & 400 & 550 & -- \\
C & 1220 & 200 & 100 & 400 & 550 & 500
\end{tabular}
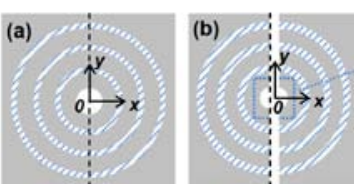

(e)

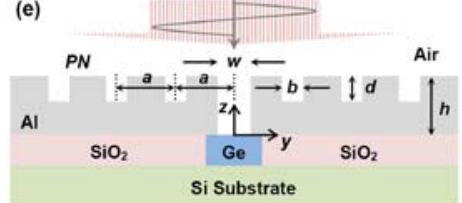

Fig. 1. Structure of the MSM Ge photodetectors.
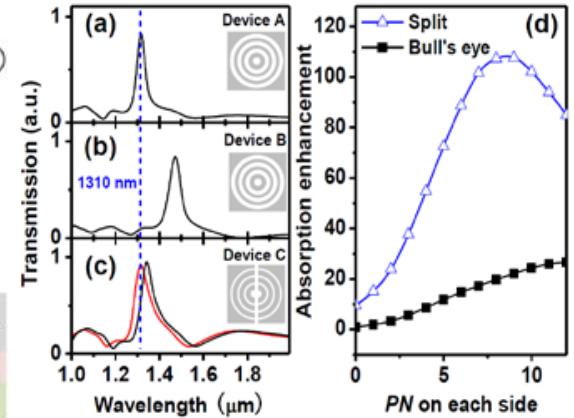

Fig. 2. (a,b,c) Numerical calculated transmission spectra of devices, and (d) the dependence of the absorption enhancement factor on $P N$ number. 
Beginning with the Helmholtz reciprocity theorem, when the light with angle $\theta$ is incident upon a metallic grating with period $a$, surface plasmon polaritons (SPPs) can be excited in accordance with the momentum conservation law $k_{\mathrm{SPP}}=k \sin \theta+m G$ [4], where, $m$ is an integer, $G=2 \pi / a$ is the reciprocal vector of the grating. For the 1 st grating order and normal incidence, the grating period can be estimated $a=\lambda / \sqrt{\varepsilon_{\mathrm{Al}} \varepsilon_{\text {air }} /\left(\varepsilon_{\mathrm{Al}}+\varepsilon_{\text {air }}\right)} \approx 1300$ $\mathrm{nm}$, where $\varepsilon_{\mathrm{Al}}$ and $\varepsilon_{\text {air }}$ represent the dielectric constants of metal and air, respectively. However, the actual value of $a$ should be slightly smaller due to Fano-type interaction. Other geometrical parameters also have influence on the SPP excitation. Assuming normal incidence and electric field parallel to y-axis, we perform the theoretical simulation on the transmission spectra of devices as shown in Fig. 2(a) to (c), respectively. By monitoring the SPP modes observed as maxima in transmittance, we can find proper but not unique structural parameters for devices as listed in Table 1. The SPP mode takes place at $1310 \mathrm{~nm}$ in devices A, but not in B owing to the non-compliance to the conservation condition. Upon directly separating the antenna of device A with $g=500 \mathrm{~nm}$, the SPP peak is redshifted by $\sim 30 \mathrm{~nm}$ (Fig. 2(c)). Thus, we reduce the period $a$ from 1290 to $1220 \mathrm{~nm}$ for device C to adjust the SPP excitation position back to $1310 \mathrm{~nm}$. Fig. 2(d) shows the absorption enhancement versus the period number $P N$ for devices $\mathrm{A}$ and $\mathrm{C}$, respectively. Here the absorption enhancement is calculated as the ratio of the absorption power of device $\mathrm{A}$ or $\mathrm{C}$ to the result from device $\mathrm{A}$ without a grating pattern. As $P N$ increases, the enhancement factor gradually increases due to an improved collection of the additional optically excited surface wave by the grating, and then saturates when additional collection of surface waves is balanced by re-radiation into photons [5]. By comparison, the absorption enhancement from device $\mathrm{C}$ (shown in blue line) is found to be much higher than that of device A (shown in black line) with the same number of $P N$. When $P N=9$, the curve of device $\mathrm{C}$ reaches saturation point and the enhancement factor is approximately 5 times that from device A, whose saturation point is located at $P N \approx 12$. It means that the split antenna with $P N=3$ could be sufficient to achieve the same absorption as the conventional bull's eye antenna with $P N=12$. As such, the entire grating diameter can be reduced greatly by $\sim 75 \%$ while maintaining the same light concentration performance as the conventional bull's eye scheme [6].

Based on the above design, we performed the fabrication of MSM Ge photodetectors A, B, and C with $P N=9$. A circular Ge region was first formed with diameter of $1 \mu \mathrm{m}$ and thickness of $200 \mathrm{~nm}$ (Fig. 3(d)) using blanked Ge epi and mesa-cut approach on an 8-inch Si wafer. Prior to the Al deposition, a passivation oxide was deposited and polished, and wet-etch to expose the Ge active region. Subsequently, the Al layer (400nm) was deposited and partially etched on the top surface to form the concentric grating. Finally, the Al layer was treated with a deep etch to form the single aperture and the additional 500-nm-wide gap for device C. Fig. 3(a) and (b), respectively, show the top-view SEM images of devices A and C with conventional and split bull's eye antennas. The cross-sectional TEM image of device $\mathrm{C}$ is shown in Fig. 3(c).

\section{Results and Discussion}

The devices were characterized using a diode laser at $1310 \mathrm{~nm}$ with an illumination power of $1 \mathrm{~mW}$. The incident light spot was focused to approximately $2.5 \mu \mathrm{m}$ in diameter through a lensed fiber. The room-temperature current-voltage $(I-V)$ characteristics from devices were measured and are shown in Fig. 4(a) and (b). With a reverse bias voltage of $2 \mathrm{~V}$, a photocurrent of $1.0 \mathrm{nA}$ was obtained from device A with SPP excitation, which is $\sim 1.5$ times of the $0.66 \mathrm{nA}$ measured using device $\mathrm{B}$. The photoresponsivity can be estimated to be 0.8 and $0.46 \mu \mathrm{A} / \mathrm{W}$ for devices A and B, respectively. Such enhancement of photoresponse is a direct experimental evidence of plasmonic effect from the Al layer in the near-infrared range [3-6]. At the same bias, a higher photocurrent of $4.6 \mathrm{nA}$ is measured from device $\mathrm{C}$ with, leading to a responsivity of $3.93 \mu \mathrm{A} / \mathrm{W}$, which is 4.9 times that of device $\mathrm{A}$ and 8.5 times that of device B. The experimental results are consistent with the theoretical prediction as described in Fig. 2(d), where the absorption enhancement from device $\mathrm{C}$ is calculated approximately 5 times that from device A at $P N$ $=9$. It indicates the superior performance of split bull's eye configuration than the conventional structure.

We performed a rigorous theoretical calculation on the electromagnetic near field using the FDTD method. Fig. $5(\mathrm{a})$ and (b) show the dynamical normalized magnetic field $(H x)$ distribution in the cross-sectional view at the plane of $x=0$ in devices A and B. Upon illumination of device A, the incident light resonantly excites SPPs on the concentric grating metal coupler, and high density optical energy is efficiently transferred by funneling SPPs through the aperture towards a central Ge active region. In comparison, the spatial distribution of optical energy in device B with a period length of $a=1450 \mathrm{~nm}$ exhibits relatively weaker intensities in the Ge area due to the absence of SPP. This direct comparison demonstrates the value of resonant plasmonic structures to concentrate optical energy into subwavelength detection region and to enhance the photoresponse and quantum efficiency. Next, we investigated the origin of additional photoresponse improvement in split bull's eye antenna. As described above, the SPPs will be generated in both devices A and C by the incident light of wavelength $1310 \mathrm{~nm}$, but the spatial 
distribution of optical near-field in the Ge region is quite different. The normalized and time-averaged $E z$-field intensity distribution around the center hole at the plane of $z=0$ is shown for devices A and C in Fig. 5(c) and (d), respectively. A much higher energy density is distributed throughout the whole Ge active area in device $\mathrm{C}$. The strong optical energy is localized in the vicinity of electrode corners, where the depletion regions are formed due to the Schottky barrier between metal $\mathrm{Al}$ and Ge material. In this case, the effective light trapping in the active region boosts the light absorption therefore generating more electron-hole pairs, thus giving rise to the improved quantum efficiency. It is expected that the overlap of high optical near-field and electric built-in field in the split bull's eye structure will result in the superior performance.

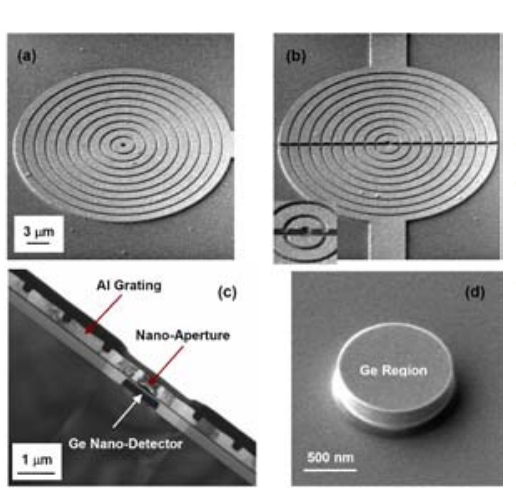

Fig. 3. (a) (b) (d) Top view SEM and (c) crosssectional TEM images of the devices.

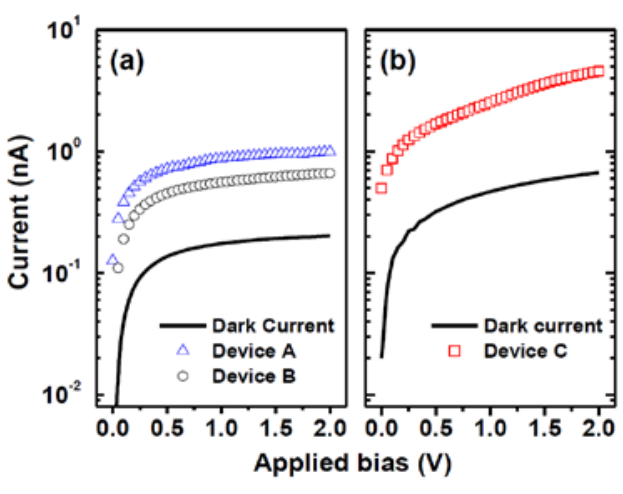

Fig. 4. Measured current-voltage (I-V) characteristics from devices.

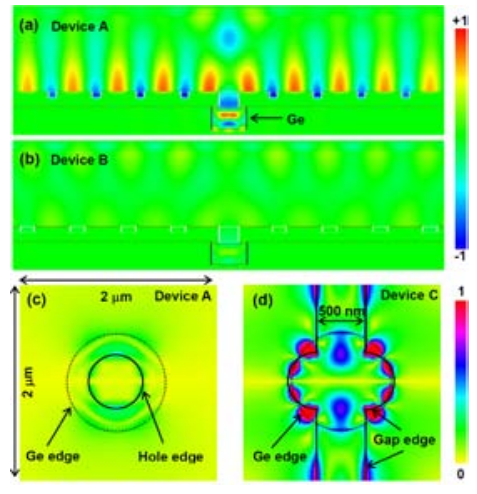

Fig. 5. FDTD-simulated optical near-field distributions of devices.

As is well known, the speed of a detector is generally limited either by its carrier-transit time or the resistancecapacitance $(R C)$ delay. At reverse bias of $1 \mathrm{~V}$, the whole Ge active region is depleted due to large the depletion width $(\sim 940 \mathrm{~nm})$ for a carrier concentration of $2 \times 10^{15} \mathrm{~cm}^{-2}$. Given a carrier saturated velocity of $6 \times 10^{6} \mathrm{~cm} / \mathrm{s}$ in Ge, the carriertransit times across the vertical (devices A and B) and lateral space (device C) are about 3.3 and $8.3 \mathrm{ps,}$ and the transit-time-limited bandwidth are 133 and $53 \mathrm{GHz}$, respectively. On the other hand, with a load resistance of $50 \Omega$, the capacitances of device A and C are estimated to be 0.192 and $0.006 \mathrm{fF}$ with the corresponding RCdelay-limited bandwidth of 16.6 and $500 \mathrm{THz}$, respectively. Therefore, the device speed is mainly limited by the transition of carriers across the depletion region in the gap. Due to relatively poor absorption in the center of gap, a reduction in the size of the gap would be expected to shorten carrier transition time and thus improve high speed response with a slight expense of reduced quantum efficiency.

\section{Conclusion}

We have theoretically and experimentally exploited the novel design of a MSM Ge photodectector that provides an excellent strategy for overcoming the inherent trade-off between quantum efficiency and speed response by using a surface plasmon antenna consisting of a split bull's eye structure and a $\Phi$-shape gap. The low-cost and CMOScompatible Al material is demonstrated to be a promising plasmonic metal candidate in the near-infrared wavelength, showing a strong capability for optoelectronic devices applications for standard communication.

\section{References}

[1] L. Tang, S. E. Kocabas, S. Latif, A. K. Okyay, D. S. Ly-Gagnon, K. C. Saraswat, and D. A. B. Miller, "Nanometre-scale germanium photodetector enhanced by a near-infrared dipole antenna," Nature Photon. 2, 226-229 (2008).

[2] T. Ishi, J. Fujikata, K. Makita, T. Baba, and K. Ohashi, "Si Nano-Photodiode with a Surface Plasmon Antenna,” Jpn. J. Appl. Phys. 44, L364L366 (2005).

[3] F. F. Ren, K. W. Ang, J. F. Song, Q. Fang, M. B. Yu, G. Q. Lo, D. L. Kwong, "Surface plasmon enhanced responsivity in a waveguided germanium metal-semiconductor-metal photodetector,” Appl. Phys. Lett. 97, 091102 (2010).

[4] S. A. Maier, Plasmonics: Fundamentals and Applications (Springer Press: New York, 2007), Chap. 2.

[5] L. Martín-Moreno, F. J. García-Vidal, H. J. Lezec, K. M. Pellerin, T. Thio, J. B. Pendry, and T.W. Ebbesen, “Theory of extraordinary optical transmission through subwavelength hole arrays,” Phys. Rev. Lett. 86, 1114-1117 (2001).

[6] F.F. Ren, K.W. Ang, J. D. Ye, M. B. Yu, G. Q. Lo, and D. L. Kwong, "Split Bull's Eye Shaped Aluminum Antenna for Plasmon-Enhanced Nanometer Scale Germanium Photodetector," Nano Lett. 11, 1289-1293 (2011). 